\title{
On-Line Automated Inspection of Poultry Carcasses by Machine Vision
}

\author{
Y.R. Chen, K. Chao, W.R. Hruschka
}

\begin{abstract}
Development of an automated poultry inspection system that is low-cost, operates with minimum human intervention, and is able to maintain its accuracy is important to the U.S. Food Safety and Inspection Service (FSIS) and the poultry industry. Such a system, placed strategically in the processing plants, would help improve the inspection speed, minimize problems of human error and variability, improve the effectiveness of the federal inspection program, and increase the slaughter plants' productivity. This paper describes an automated poultry inspection system that utilizes machine vision technology. The system was tested on-line at a poultry plant. The results showed that the classification accuracy for wholesome and unwholesome carcasses were 94 and $87 \%$, respectively.
\end{abstract}

Keywords: Mulitspectral, Dual-Camera, Slaughterhouse, Carcasses, High-speed-processing

\section{Introduction}

Poultry consumption in the United States has increased in recent years. Per capita consumption of chicken increased from 33.3 pounds in 1965 to 82.0 pounds in 1996, and reached 91 pounds in 1999. The number of chickens slaughtered at U.S. federally inspected establishments was 7.9 billion birds in 1997, valued at 14.1 billion dollars (USDA, 1999). Presently, each chicken intended for sale to U.S. consumers is required by law to be inspected post-mortem by a United States Department of Agriculture (USDA) Food Safety and Inspection Service (FSIS) inspector for its wholesomeness (USDA, 1984). These inspectors visually and manually inspect poultry carcasses and viscera on-line at processing plants. However, the organoleptic inspections are based on inspectors' professional judgments, which in turn are based on what they see, feel, and smell. Although USDA has made adjustments, changes, and modifications over the years in an effort to keep abreast of the ever-changing poultry industry, the traditional organoleptic methods of inspection have not been changed much.

There is an urgent need to develop automated inspection systems that can operate on-line in real-time (at least 140 birds per minute) in the slaughter plant environment. These systems should be able to accurately detect and identify carcasses with infectious condition, particularly septicemia/toxemia (septox), and fecal contamination. They should also detect animal diseases, particularly airsacculitis, ascites, cadaver, and inflammatory process (IP), and other defects such as bruises,

1 Instrumentation and Sensing Laboratory, USDA, cheny@ba.ars.usda.gov 
tumors, sores, and scabs. Low-cost automated machine vision inspection systems, placed strategically in the processing plants, would help improve the inspection speed, minimize problems of human error and variability, improve the effectiveness of the federal inspection program, and increase the slaughter plants' productivity.

Since 1991, at the Instrumentation and Sensing Laboratory (ISL), we have been conducting research to develop automated poultry inspection systems that can operate on-line in real-time (at least $140 \mathrm{bpm}$ ) in the slaughter plant environment. We have successfully developed and tested two systems in a slaughterhouse of a Tyson Poultry Plant in New Holland, Pennsylvania, in the past years. One system employs the visible/near-infrared technology, and the other utilizes a multiple camera machine vision technique. This paper presents the development and testing of the automated poultry inspection system that utilizes machine vision technology.

\section{Mulitspectral Imaging Techniques}

Many applications using machine vision technology have been developed in the agricultural sector, such as land-based and aerial-based remote sensing for natural resources assessments, precision farming, postharvest product quality and safety detection, classification and sorting, and process automation. Advantages of using imaging technology for sensing are that it can be fairly accurate, non-destructive, and yields consistent results.

An intensified multispectral imaging system was used to discriminate wholesome poultry carcasses from unwholesome carcasses (Park and Chen, 1994). Based on the studies by Chen and Massie (1993), the wavebands of the six optical filters of $542,570,641,700,720$, and $847 \mathrm{~nm}$ were chosen. The system showed an accuracy of $89.3 \%$ for separation of wholesome carcasses from unwholesome carcasses. For separation of wholesome carcasses from septicemia, the accuracy of classification was also $89.3 \%$, and separation accuracy from cadaver was $96.5 \%$.

The textural features based on co-occurrence matrix (COM) of multispectral images of poultry carcasses were analyzed for discriminating unwholesome poultry carcasses from wholesome carcasses (Park, et al., 1998). It was found that the wholesome carcasses scanned at both $542 \mathrm{~nm}$ and $700 \mathrm{~nm}$ wavelengths were significant parameters for separating septicemic and cadaver carcasses.

\section{A Transportable Dual-Camera System}

Based on the above study (Park, et al., 1998), a transportable dual-camera system, using filters of 542 and $700 \mathrm{~nm}$, was assembled using industrial machine frames (ParFrame, Parker automation, Wadsworth, OH) (Chao, et al., 2000). A schematic of the dual-camera system is shown in Figure 1. The description of its major components is given in Chao, et al. (2000), where a laboratory version of the system is described. In the transportable system, two fiber-optic dual-line lights (QDF5048, Dolan-Jenner Industries, Inc., Lawrence, MA) equipped with AC regulated $150 \mathrm{~W}$ quartz-halogen were used to provide evenly distributed illumination to the poultry carcasses. Two adjustable hinges were mounted at the end of two extended profiles as pivoting joints to provide free rotation to the dual-line lights. The dual-line lights 
were positioned bilaterally at $45^{\circ}$ angles to provide balanced area illumination to the poultry carcass.

For this machine vision inspection system (MVIS), object-oriented programming paradigms (Rumbaugh, et al., 1991) were utilized to integrate the hardware components. The system performs real-time imaging acquisition, processing, and on-line classification of poultry carcasses.

The image processing is performed in real-time (two images per chicken, at 540 and $700 \mathrm{~nm}$ ). The image is reduced to a size of $256 \times 240$ pixels and then segmented from the background using simple thresholding. A total of 15 horizontal layers (16 horizontal lines of pixels each) are generated from each segmented image, as shown in Figure 2. For each layer, a centroid is calculated from the binarized image. Based on these centroids, each layer was divided into several square blocks (16x16 pixels), for a total of 107 blocks. The averaged intensity of each block is used as the input data to the neural network models. The constant number of blocks in each layer was previously determined to delineate the main part of each carcass and omit the legs and wings. Note that for a very small chicken, the edge blocks could contain several background pixels, passing chicken size information on to the neural net in the form of lowered average intensity.

Model development was conducted off-line from images acquired on-line. When acquiring images for off-line model development, a stainless steel plate holding a magnet is hung on each shackle suspending a chicken carcass, marking it for image acquisition and indicating the condition of the chicken. The position of the magnet (high or low) indicates whether the chicken is wholesome or unwholesome, respectively. In this mode, the veterinarian marks (by hanging the appropriate magnetic markers) those chickens that provide a wide range of size and appearance within each class (wholesome and unwholesome).

After off-line development of the backpropagation neural network models, parameters (including weights and biases from the optimized neural network models) are saved in the ASCII data format. These parameters are then incorporated into the on-line classification section of the MVIS software. When conducting on-line testing, a photoelectric proximity sensor triggers image capture when a shackle is sensed. In this mode, images of all carcasses are captured, and the veterinarian marks only the unwholesome chickens by hanging the stainless steel plate with the lower magnetic marker. Immediately following the on-line image processing, one-pass forward mapping of the neural network application is performed to classify the carcasses as wholesome or unwholesome.

The dual-camera system was used to inspect chicken carcasses at a processing plant in Pennsylvania over a 14-day period in September of 1999 (Chao, et al. 2001). The dual-camera system was installed between the evisceration station and inspector station. Table 1 summarizes the number of carcasses that were acquired for model development and on-line testing of the inspection system. A total of 1,400 poultry carcasses (700 wholesome and 700 unwholesome) were measured for development of classification models. The 'first' data set used all 1,000 samples collected on or before 9/20/99. The 'second' data set used the first data set plus 300 samples (150 wholesome and 150 unwholesome) from the data collected 9/21-22/99. The 'third' 
data set consists of the second data set plus 100 samples (50 wholesome and 50 unwholesome) from the data collected on 9/27/99. This procedure progressively widened the variability of the calibration sets and thereby the predictive ability of the models developed from them.

A feed-forward-back-propagation neural network model with 107 input nodes, 10 nodes in one hidden layer, and 2 output nodes, was used. The output nodes' target outputs are $\left(\begin{array}{ll}0 & 1\end{array}\right)$ or $\left(\begin{array}{ll}1 & 0\end{array}\right)$ depending on whether the sample was identified wholesome or unwholesome by the veterinarian. For each of the three data sets, model development method starts with splitting the data into two sub-sets: training (50\%) and validation $(50 \%)$. Each sub-set contains equal numbers of wholesome and unwholesome carcasses. The neural network models are trained on the training sub-set. The validation sub-set is used to decide which network model and how much training is optimal. The validation set is predicted every 200 iterations of the training cycle, and the network weights are saved if the classification results have been improved. Training is always stopped after 15,000 iterations.

A total of 13,191 poultry carcasses (12,432 wholesome and 759 unwholesome). In each case, the 540 and $700 \mathrm{~nm}$ results were combined using an AND operation to give a single prediction. That is, a carcass is predicted wholesome only if the data from both cameras result in wholesome prediction.

For each of the three data sets, four neural network models were tested to select the optimum for use in subsequent on-line classification of poultry carcasses. Models 1 and 2 utilized the same delta learning rule, but different transfer functions. Model 1 and Model 2 used hyperbolic tangent and sigmoid transfer functions, respectively. Models 3 and 4 ulitilized norm-cum-delta learning rule, but with hyperbolic tangent transfer function for Model 3, and sigmoid transfer function for Model 4. With some exceptions, all models and data sets showed similar results: about $95 \%$ classification accuracy for wholesome, $88 \%$ for unwholesome, and $93 \%$ for combined. The 700 $\mathrm{nm}$ results were slightly less accurate than those of the $540 \mathrm{~nm}$ for unwholesome. We suspect that this is because the more useful information is contained in the myoglobin absorbing area near $540 \mathrm{~nm}$.

There was some improvement from adding the 300 samples to the first data set to form the second data set, but little when adding the last 100 samples to make the third data set. This indicates that during the time of the experiment, 1300 samples were sufficient to define the variability. It is probable that more data would need to be added in a longer-term test, when new populations of chickens (different breeds, feeding regimens, seasons, etc.) are encountered.

As in previous work (Chao, et al., 2000), there were no striking differences between the models. Our choice of the model to use in subsequent online testing is based on the validation performance on 'all carcasses', with ties going to model 1 . Thus we used model 1 throughout for the $700 \mathrm{~nm}$ data; and we used model 2 for the $540 \mathrm{~nm}$ data, except for the first data set, where the slightly better performance on the unwholesome carcasses prompted a decision for model 1.

Table 2 shows the on-line classification results using the six best models discussed above. As stated above, the 540 and $700 \mathrm{~nm}$ results were combined using an AND 
operation to give a single prediction. This gave three sets of results: the first when the models developed from the first data set were tested on-line on 9/22/99, the second when the models developed from the second data set were tested on-line on 9/23-24/99, and the third when the models developed from the third data set were tested on-line on 9/28-30/99.

In each case the results for the wholesome were better than those for the unwholesome, as was seen in the model development stage. The results improved from sets 1 to 2 as in model development. They also improved from set 2 to 3 , indicating that the expansion of the variability in the model development sets was more than could be detected at the model development stage. We would expect the on-line testing results to be less accurate than those for training, partly because that is usually the case in a training/testing situation, and partly because of the use of the AND function. But in the third trial, the results for the wholesome improved to the 94\% classification accuracy, and the unwholesome classification reached the $87 \%$ classification accuracy. This shows that the broadening of the variability in the third training set was useful in on-line predicting, even though it was not detected in the training stage.

\section{Summary}

An automated inspection system should be low-cost, operate with minimum human intervention, and be able to maintain its accuracy. The Instrumentation and Sensing Laboratory (ISL) has successfully applied multispectral imaging technologies and developed an automated system for on-line inspection of poultry carcasses at slaughter plants. Trials for the on-line poultry inspection system at a commercial slaughter facility showed that the ISL automated inspection system can be implemented on poultry slaughter lines to separate unwholesome from wholesome chicken carcasses. The results showed that, for the wholesome improved to the 94\% classification accuracy of the training stage, and the unwholesome classification reached the $87 \%$ classification accuracy of the training stage. Such a system, placed strategically in poultry slaughter plants, would help increase throughput of products, minimize problems of human inspection error and variability, improve the effectiveness of the federal inspection program, and ease consumer concerns about the safety of USDA-inspected poultry and poultry products.

\section{References}

Chao, K., B. Park, Y.R. Chen, W.R. Hruschka, F.W. Wheaton. 2000. Design of a dual-camera system for poultry carcasses inspection. Applied Engineering in Agriculture. 16(5):581-587.

Chao, K., Y.R. Chen, W.R. Hruschka. 2001. On-line inspection of poultry carcasses by dual-camera system. Submitted to Journal of Food Engineering. (In review).

Chen, Y.R., and D.R. Massie. 1993. Visible/Near-Infrared Reflectance and Interactance Spectroscopy for Detection of Abnormal Poultry Carcasses. Trans. ASAE. 36(3):863-869. 
Park, B. and Y.R. Chen. 1994. Intensified Multispectral Imaging System For Poultry Carcass Inspection. Transaction of the ASAE. 37(6): 1983-1988. Nov./Dec.

Park, B., Y.R. Chen, and K. Chao. 1998. Multispectral imaging for detecting contamination in poultry carcasses. In: SPIE Proceedings on Pathogen Detection and Remediation for Safe Eating. Y.R. Chen, ed., Boston, Massachusetts. 3544:156-165.

Rumbaugh, J., M. Blaha, W. Premerlani, F. Eddy, and W. Lorensen. 1991. Object-oriented modeling and design. Chapter 19. pp. 417-432. Prentice-Hall, Inc., Englewood Cliffs, New Jersey.

USDA National Agricultural Statistics Service. 1999. Agricultural Statistics 1999. Washington, D.C.

USDA. 1984. A review of the slaughter regulations under the Poultry Products Inspection Act. Regulations Office, Policy and Program Planning, FSIS, USDA, Washington, D.C.

USDA National Agricultural Statistics Service. 1999. Agricultural Statistics 1999. Washington, D.C.

USDA. 1984. A review of the slaughter regulations under the Poultry Products Inspection Act. Regulations Office, Policy and Program Planning, FSIS, USDA, Washington, D.C.

\section{Tables}

Table 1 - Number of carcasses used for model development and on-line testing.

\begin{tabular}{|c|c|c|c|c|}
\hline Date Collected & Wholesome & Unwholesome & Data Set & Total \\
\hline 9/16/99 - 9/20/99 & 500 & 500 & First & 1,000 \\
\hline $9 / 21 / 99-9 / 22 / 99$ & 150 & 150 & Second & 1,300 \\
\hline $9 / 27 / 99$ & 50 & 50 & Third & 1,400 \\
\hline Total & 700 & 700 & & \\
\hline \multicolumn{5}{|l|}{ On-line testing } \\
\hline & & & \multicolumn{2}{|c|}{ Using models developed from } \\
\hline $9 / 22 / 99$ & 2,049 & 124 & \multicolumn{2}{|c|}{ First data set } \\
\hline 9/23/99 - 9/24/99 & 4,431 & 240 & \multicolumn{2}{|c|}{ Second data set } \\
\hline 9/28/99 - 9/30/99 & 5,952 & 395 & \multicolumn{2}{|l|}{ Third data set } \\
\hline Total & 12,432 & 759 & & \\
\hline
\end{tabular}


Table 2 - Classification accuracy for on-line testing.

\begin{tabular}{|c|c|c|c|c|}
\hline Test on & & & Predicte & \\
\hline$\underline{\operatorname{Day}(\mathrm{s})}$ & & Wholesome & Unwholesome & Accuracy $(\%)$ \\
\hline- & Wholesome & 1864 & 185 & 90.9 \\
\hline 9/22/9 Act & & & & \\
\hline $9 \quad$ al & Unwholesome & 22 & 102 & 82.2 \\
\hline 9/23/9 & Wholesome & 4126 & 305 & 93.1 \\
\hline 9-9/24/ Act & & & & \\
\hline $99 \quad$ al & Unwholesome & 34 & 206 & 85.8 \\
\hline $9 / 28 / 9$ & Wholesome & 5599 & 353 & 94.0 \\
\hline 9-9/30/ Act & & & & \\
\hline $99 \quad \mathrm{al}$ & Unwholesome & 50 & 345 & 87.3 \\
\hline
\end{tabular}




\section{Figures}

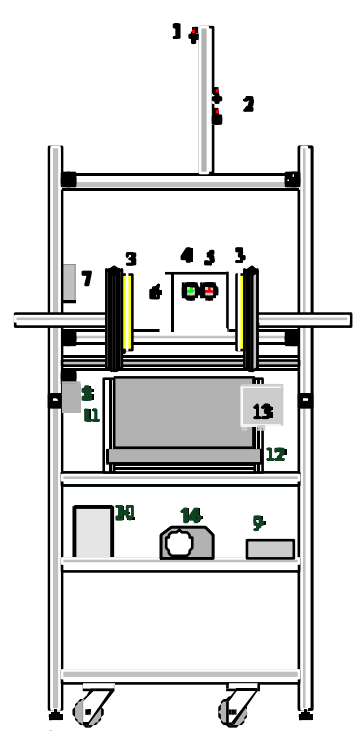

Front View

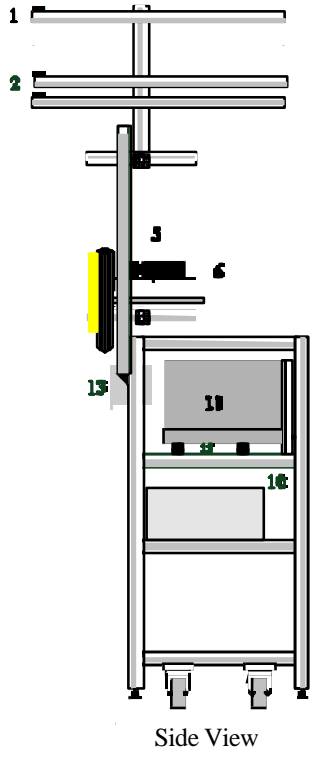

1. photoelectric sensor

2. magnetic sensor

3. quartz halogen line liq

4. camera with $700 \mathrm{~nm}$

5. camera with $540 \mathrm{~nm}$

6. camera enclosure

7. indicator and stop swi

8. interconnector for sen

9. power supply

10. battery backup

11. industrial computer (200 MHz)

12. keyboard

Figure 1 - Schematic diagram of the 2-camera system for poultry inspection

(a)
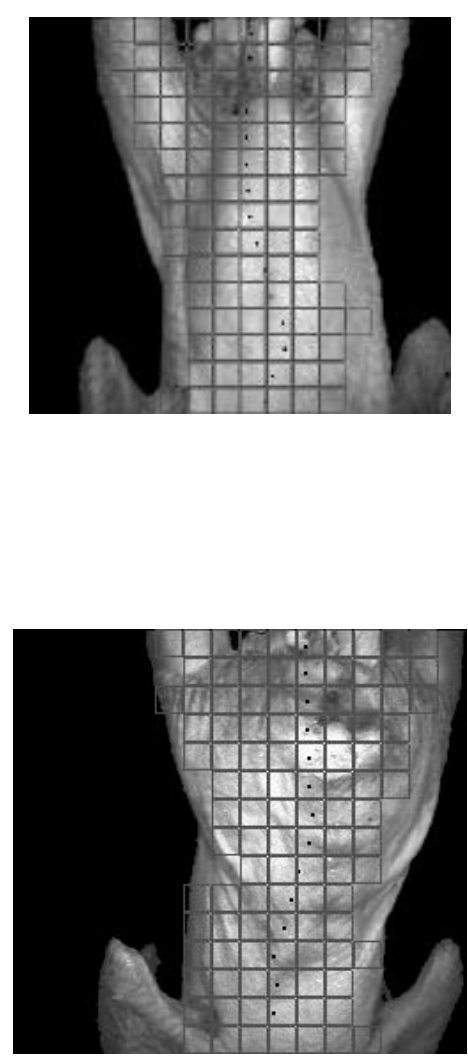

(c) (b)
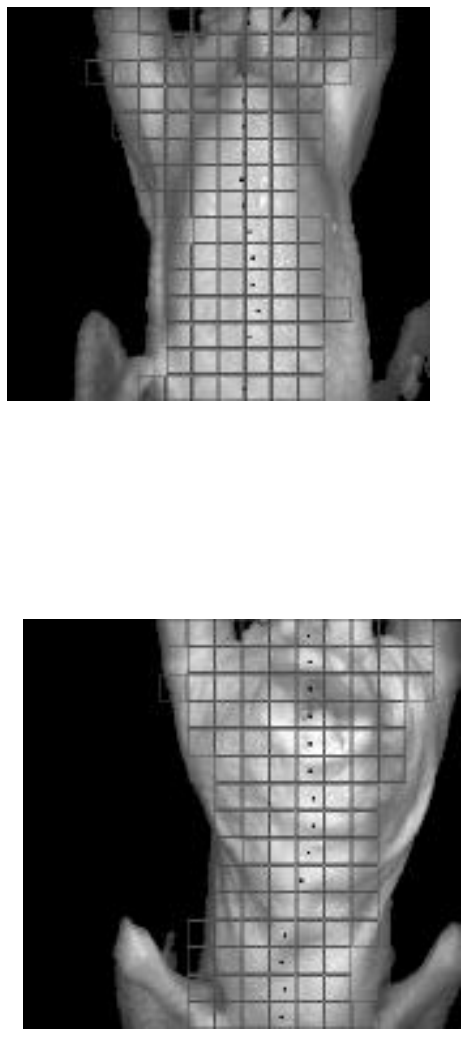

(d)

Figure 2 - Real-time image processing from the MVIS. Centroid and mesh generation during image capture for off-line training. (a) front at $540 \mathrm{~nm}$, (b) front at $700 \mathrm{~nm}$, (c) back at $540 \mathrm{~nm}$, (d) back at $700 \mathrm{~nm}$. 